\title{
Análisis de la aceptabilidad, consumo y aporte nutricional del programa alimentario del adulto mayor
}

\author{
Celia Masi, Eduardo Atalah
}

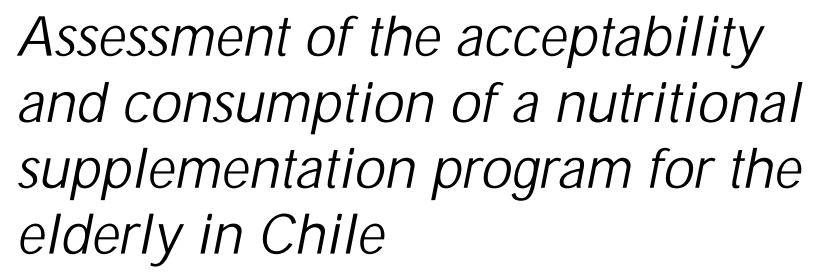

Background: During 2005, the Chilean Ministry of Health included a fortified milk beverage to the Elderly Food Supplementation Program, to improve the compliance with the program and to increase the provision of micronutrients. This beverage was added to a soup that was distributed since 1999. Aim: To evaluate the long term acceptability, tolerance and consumption of the Milk Beverage and analyze the nutritional contribution of the products provided by the supplementation program to the usual diet of the elderly. Material and methods: One hundred twenty five elderly subjects, aged $77 \pm 4$ (52 males) living in Metropolitan Santiago, aged over 70 years, beneficiaries of the food supplementation program, were interviewed. The acceptability, consumption and tolerance of the Milk Beverage were evaluated by specially designed surveys. The contribution of the food suplementation products to daily nutrient intake was also analyzed by 24 hours dietary recalls. Results: The mean body mass index of the interviewed elderly subjects was $27.0 \pm 4.5 \mathrm{Kg} / \mathrm{m}^{2}$. Monthly delivery of the products included in the program increased significantly after the incorporation of the Milk Beverage to the program. The milk beverage had a significantly higher daily consumption and a lower family dilution than the soup. The consumption of supplementation products increased significantly the daily intake of energy and most micronutrients. Noteworthy was the case for vitamin $\mathrm{B}_{12}, \mathrm{C}, \mathrm{E}$ and zinc, whose intake doubled ( $\mathrm{p}<0.01$ ). Conclusions: The Milk Beverage had an excellent approval and consumption rate. The consumption of food supplementation products improved daily intake of most nutrients in elderly subjects (Rev Méd Chile 2008; 136: 415-22).

(Key w ords: Aged; Dietary supplements; Milk)

Recibido el 2 de agosto, 2007. Aceptado el 13 de noviembre, 2007.

Departamento de Nutrición, Facultad de Medicina, Universidad de Chile. Santiago de Chile.

${ }^{a}$ Nutricionista, Magíster en Cs. Biológicas Mención Nutrición, Universidad de Chile.

Correspondencia a: Dr. Eduardo Atalah S. Departamento de Nutrición, Facultad de Medicina, Universidad de Chile. Independencia 1027, 2o P. Fax: 737-8778.

E mail: eatalah@med.uchile.cl 
$\mathrm{E}^{\mathrm{n}}$ n las últimas décadas, la tasa de natalidad ha disminuido y la esperanza de vida ha aumentado considerablemente en la población chilena, lo que ha determinado cambios significativos en la pirámide poblacional. Al comparar los últimos censos de población, se observa que los menores de 15 años crecieron a un promedio anual de $0,5 \%$, mientras que los adultos mayores lo hicieron a un ritmo 5 veces mayor (2,7\% anual). Actualmente, las personas mayores de 70 años representan $4,4 \%$ de la población nacional, porcentaje que aumentará a casi el doble $(8,2 \%)$ para el año $2025^{1,2}$.

Más de 500.000 adultos mayores en el país reciben pensiones asistenciales o de ancianidad que no superan los $\$ 100.000$ mensuales y en muchos casos inferiores a \$50.000. Las limitaciones económicas en que viven muchos adultos mayores, junto con las alteraciones psicológicas, sensoriales y metabólicas que se producen a esta edad, determinan que una fracción importante de ellos reciba una alimentación deficiente. Un estudio realizado en Chile a fines de la década 199099 mostró que la alimentación de los adultos mayores de bajos recursos presenta importantes deficiencias, especialmente de calcio, zinc, ácido fólico, vitaminas $\mathrm{A}, \mathrm{C}$ y $\mathrm{B}_{12}$, con muchas más carencias que los ancianos de ingresos medios ${ }^{3-5}$. Ello incentivó al Ministerio de Salud a crear, en 1999, un programa alimentario orientado a este grupo de población, con el fin de mejorar su estado nutricional, la funcionalidad y su calidad de vida.

El Programa de Alimentación Complementaria para el Adulto Mayor (PACAM), se inició con la distribución de dos kilos mensuales de un alimento precocido llamado "Crema Años Dorados", elaborado con harina de leguminosas y cereales y una mezcla de vitaminas y minerales ${ }^{6}$. Dado que las raciones recomendadas son de $50 \mathrm{~g}$, se podrían consumir dos raciones diarias del producto, durante 20 días al mes. Una evaluación inicial demostró buena aceptabilidad por parte de los beneficiarios, pero después de algunos años su consumo aparentemente disminuyó por la fatiga que determina el uso repetido de un producto de un sabor muy definido. Ello se intentó superar con la incorporación de nuevos sabores, aunque según la impresión de los equipos de atención primaria, el consumo real seguía siendo menor de lo esperado.
Por esto el Ministerio de Salud decidió explorar nuevas opciones de suplementación alimentaria, desarrollando en conjunto con el Departamento de Nutrición de la Facultad de Medicina de la Universidad de Chile una bebida láctea, alimento que puede ser consumido diariamente y que podría aumentar la ingesta global de nutrientes por parte de los adultos mayores. Un estudio preliminar de un prototipo mostró excelente aceptabilidad y tolerancia en un periodo corto de tiempo (15 días), por lo cual se decidió su utilización a nivel nacional, a partir de agosto de 2005.

La "Bebida Láctea Años Dorados" (BLAD) es un producto alimentario en polvo, elaborado con base de leche y cereales con un suave sabor a vainilla. Es instantáneo, reducido en lactosa, bajo en grasa y en sodio, fortificado con vitaminas y minerales en función de las necesidades de los adultos mayores. Se prepara diluyendo una porción de $25 \mathrm{~g}$ de polvo en $200 \mathrm{ml}$ de agua, la que aporta $102 \mathrm{Kcal}$, 4,5 g de proteínas y 20\% o más de las necesidades diarias de muchos micronutrientes (Vitaminas C, D, E, B 12 , calcio, etc.) ${ }^{7}$.

Desde la introducción de la "Bebida Láctea Años Dorados", no existen datos sobre la opinión que la población beneficiaria tiene de los productos distribuidos por el programa. Tampoco existen estudios que analicen el aporte que realiza a la alimentación del adulto mayor. Para ello, se realizó este estudio cuyo propósito fue evaluar la aceptabilidad, tolerancia y consumo de los alimentos del programa y el aporte de los mismos a la alimentación diaria en una muestra de beneficiarios del PACAM de distintos consultorios de la Región Metropolitana.

\section{MATERIAL Y MÉTODOS}

Diseño. Se realizó un estudio transversal analítico en una muestra de adultos mayores beneficiarios del programa PACAM de la Región Metropolitana. Los criterios de inclusión fueron: edad 70 a 85 años, autovalente, mentalmente competente para responder las encuestas y al menos 18 meses de permanencia en el programa. Se excluyeron a personas que presentaban enfermedades crónicas graves (cáncer, insuficiencia renal, diabetes avanzada, etc.) o que se hubieran incorporado al programa menos de 18 meses. Se seleccionaron por conveniencia 4 
consultorios de atención primaria de la ciudad de Santiago: Agustín Cruz Melo (Independencia), Dr. Norman Voullieme (Cerrillos), Maipú (Maipú) y Alejandro del Río (Puente Alto).

Tamaño de la muestra. Se estudiaron dos grupos independientes: uno para analizar aceptabilidad y consumo habitual de los alimentos del programa y otro para evaluar el aporte nutricional de los productos del programa a la alimentación diaria. Para el estudio de aceptabilidad se consideró un consumo promedio semanal de 4,5 $\pm 2,0$ días para la crema, un aumento de 1,5 días con la bebida láctea, nivel de significación de 0,05 , poder $90 \%$ y efecto de diseño 1,2, lo que determinó estudiar 47 personas por grupo. Para la encuesta alimentaria se consideró $80 \pm 40 \%$ de adecuación a las recomendaciones nutricionales en personas que no consumieron alimentos del PACAM, $110 \pm 55 \%$ de adecuación en las personas que recibieron alimentos del programa, nivel de significación 0,05 , poder $90 \%$ y efecto de diseño de 1,2 , lo que determinó la necesidad de estudiar 48 personas por grupo.

Encuesta de aceptabilidad. Se elaboró una encuesta con preguntas redactadas lo más claramente posible, con un lenguaje simple y comprensible, para la población en estudio. Las principales variables fueron: características sociodemográficas, datos antropométricos, presencia de enfermedades crónicas, frecuencia, cantidad y preparación de los alimentos del PACAM, tiempo de permanencia en el programa, frecuencia de retiro de los alimentos, características organolépticas y apreciación global de los alimentos del programa. La tolerancia a los alimentos fue medida a través de preguntas acerca de la presencia de síntomas ante el consumo de la sopa crema o la "Bebida Láctea Años Dorados" tales como cólicos, vómitos, diarrea, meteorismo o constipación. Previo a la aplicación del instrumento de medición, éste fue validado mediante una prueba piloto llevada a cabo en una muestra con las mismas características de la población a estudiar.

Encuesta alimentaria. Se aplicó una encuesta alimentaria de recordatorio de $24 \mathrm{~h}$, con el fin de estimar el consumo actual de alimentos y el aporte nutricional de los productos del programa PACAM en esta población. La aplicación de la encuesta alimentaria tuvo una duración de aproximadamente 25 min. Para el análisis de los datos de dichas encuestas se utilizó el programa computacional Food Processor II (FP2), a través del cual fueron calculadas las cantidades de macro y micronutrientes aportados por la alimentación general y por los alimentos distribuidos por el PACAM.

Las cantidades de alimentos consumidos fueron convertidas en gramos considerando las porciones de intercambio y composición química de los alimentos de la pirámide alimentaria chilena. Para el cálculo de adecuación de la ingesta energética, se estimó el metabolismo basal utilizando las ecuaciones propuestas por el Comité FAO/OMS/UNU 2004, teniendo en cuenta la edad, sexo, peso y un factor de actividad 1,3 (actividad sedentaria), lo que determinó valores promedio de 1.970 y 1.736 Kcal para hombres y mujeres, respectivamente ${ }^{8}$.

Para el cálculo de adecuación de la ingesta proteica, se estimó $1 \mathrm{~g}$ de proteína por kilogramo de peso, (IOM, Dietary Reference, 2002), lo que determinó una recomendación promedio de $70 \mathrm{~g}$ de proteínas en hombres y $64 \mathrm{~g}$ en mujeres. La adecuación de la ingesta de micronutrientes se calculó en base a las recomendaciones actuales para mayores de 60 años de la Food and Nutrition Board del Instituto de Medicina de los Estados Unidos de Norteamérica?.

Aprobación por Comité de Ética. Por las características del estudio, no se consideró indispensable aprobación de un Comité de Ética Institucional.

Estadística. Los datos fueron procesados en el programa Stata 9.2, analizándose en primer lugar la distribución de cada variable. En las variables con distribución normal se utilizaron promedios y desviaciones estándar y en las variables que no se ajustaron a ella, mediana y percentiles. La comparación entre grupos de las variables continuas se hizo por medio del test de Kruskal Wallis. En las variables nominales y categóricas se analizaron la distribución de frecuencia y $\chi^{2}$. Se consideraron como significativas las diferencias con un valor de $\mathrm{p}<0,05$. 


\section{Resultados}

En el estudio de aceptabilidad se estudiaron 125 personas (52 hombres y 73 mujeres), con edad promedio de 77,3 \pm 4,3 años, la mayor parte de ellos con educación básica incompleta. El número promedio de personas del hogar fue 2,9 $\pm 2,0$, aunque $25 \%$ de la población en estudio vivía solo/a (Tabla 1). El promedio de IMC fue $27,0 \pm 4,5 \mathrm{Kg} / \mathrm{m}^{2}$, significativamente mayor en mujeres $(\mathrm{p}<0,05)$. El tiempo promedio de participación en el PACAM era $46 \pm 23$ meses, y un tercio de los beneficiarios había recibido alimentos por 5 años o más. Se observó una alta prevalencia de enfermedades crónicas, como hipertensión arterial $(82,4 \%)$, diabetes mellitus (27\%) u otras enfermedades como dislipidemia, osteoporosis, artritis, artrosis, glaucoma o cataratas.
En los seis meses previos al estudio, los beneficiarios retiraron alimentos 5,2 \pm 1,2 veces, cifra significativamente mayor que la observada en los mismos meses, el año anterior, antes de la introducción de la bebida láctea al programa (4,6 \pm 1,9 meses, p <0,001). La evaluación de las características organolépticas de la bebida láctea, en general, fue positiva, con una nota promedio superior a 6 en olor, sabor y textura.

La frecuencia de consumo semanal, el número de raciones diarias y la cantidad consumida por vez de ambos alimentos, se muestra en la Tabla 2. La frecuencia de consumo de la bebida láctea por el beneficiario fue significativamente mayor que la "Crema Años Dorados", lo que se refleja en una menor dilución intrafamiliar. El 80\% de la población estudiada manifestó consumir diariamente la BLAD, proporción que disminuyó a $31 \%$ en el

Tabla 1. C aracterísticas generales de la población estudiada

\begin{tabular}{|lcccc|}
\hline & $\begin{array}{c}\text { Hombres } \\
\mathrm{n}=52\end{array}$ & $\begin{array}{c}\text { Mujer } \\
\mathrm{n}=73\end{array}$ & $\begin{array}{c}\text { Total } \\
\mathrm{n}=125\end{array}$ & $\mathrm{P}$ \\
\hline Edad (años) & $77,2 \pm 4,7$ & $77,4 \pm 4,1$ & $77,3 \pm 4,3$ & $\mathrm{NS}$ \\
Peso (K) & $71,2 \pm 11,7$ & $62,3 \pm 13,9$ & $66,3 \pm 13,6$ & $\mathrm{NS}$ \\
Talla (m) & $1,65 \pm 0,1$ & $1,49 \pm 0,2$ & $1,56 \pm 0,2$ & $<0,001$ \\
IMC $\left(\mathrm{Kg} / \mathrm{m}^{2}\right.$ ) & $26,0 \pm 3,4$ & $27,6 \pm 5,0$ & $27,0 \pm 4,5$ & $<0,05$ \\
Meses en el PACAM & $45,4 \pm 23,6$ & $47,0 \pm 22,0$ & $46,3 \pm 22,6$ & NS \\
Personas en el hogar & $3,3 \pm 1,9$ & $2,7 \pm 2,1$ & $2,9 \pm 2,0$ & $\mathrm{NS}$ \\
\hline
\end{tabular}

Tabla 2. C antidad, frecuencia de consumo y dilución intrafamiliar de los alimentos del PACAM

\begin{tabular}{|lcrr|}
\hline & $\begin{array}{c}\mathrm{CAD}^{1} \\
\text { Promedio } \pm \mathrm{DE}\end{array}$ & $\begin{array}{c}\mathrm{BLAD}^{2} \\
\text { Promedio } \pm \mathrm{DE}\end{array}$ & $\mathrm{p}$ \\
\hline Consumo semanal (días/semana) & $4,5 \pm 2,1$ & $6,2 \pm 1,6$ & $<0,001$ \\
Consumo diario (veces/día) & $1,4 \pm 0,7$ & $1,7 \pm 0,7$ & $<0,001$ \\
Cantidad consumida por vez \\
(\% de la porción recomendada) & $104 \pm 53$ & $106 \pm 47$ & $\mathrm{NS}$ \\
Dilución intrafamiliar (\%) & 52,0 & 28,0 & $<0,001$ \\
\hline
\end{tabular}

${ }^{1}$ Crema Años Dorados.

${ }^{2}$ Bebida Láctea Años Dorados 
caso de la sopa. En ambos productos, la cantidad utilizada por ración, fue muy cercana a la recomendación (50 y $25 \mathrm{~g}$, respectivamente).

El aporte global de la alimentación, en las personas que consumieron o no alimentos del PACAM el día anterior de la encuesta, se presenta en la Tabla $3(\mathrm{n}=100)$. La incorporación de los alimentos del programa determinó un aumento significativo del aporte de energía y de prácticamente todos los micronutrientes, destacando la vitamina $\mathrm{B}_{12}$, $\mathrm{C}$, E y zinc, que aumentaron más del doble.

La misma información, pero en función del porcentaje de adecuación a las recomendaciones, se presenta en la Tabla 4. Se observa una baja adecuación de energía, proteínas, fibra, vitamina A y calcio. La adecuación de todos los micronutrientes aumentó significativamente cuando la alimentación fue complementada, especialmente en el caso de las vitaminas $\mathrm{B}_{12}$, C, E, zinc y calcio.

La Tabla 5 muestra el porcentaje de energía y nutrientes que aportaron los alimentos del PACAM a la alimentación diaria, consumidos en forma aislada o cuando se incluyen ambos productos. El aporte promedio fue especialmente relevante en calcio, hierro, vitaminas A, C y $\mathrm{B}_{1}$, representando más de $25 \%$ de la ingesta diaria. El aporte de micronutrientes a la alimentación general fue mayor en la bebida láctea que la crema y cuando se incorporaron ambos productos se lograron aportes superiores a $50 \%$ en varios nutrientes.

\section{DisCUSIÓN}

Una de las mayores dificultades de los programas de alimentación complementaria es determinar el alimento más adecuado para mejorar el aporte nutricional, presente buena tolerancia, bajo nivel de rechazo o fatiga por consumo crónico y baja dilución intrafamiliar ${ }^{10-12}$. Los alimentos tradicionales (arroz, fideos, legumbres, leche, etc) tienen la gran ventaja de una mejor aceptabilidad, pero el aporte de micronutrientes es bajo y presentan

Tabla 3. M ediana del aporte de energía y nutrientes de la alimentación complementada o no con PACAM

\begin{tabular}{|c|c|c|c|c|c|}
\hline \multirow[t]{2}{*}{ Nutrientes } & \multicolumn{2}{|c|}{$\begin{array}{l}\text { Alimentación sin } \\
\operatorname{PACAM}^{1}(\mathrm{n}=27)\end{array}$} & \multicolumn{2}{|c|}{$\begin{array}{l}\text { Alimentación con } \\
\operatorname{PACAM}^{1}(\mathrm{n}=73)\end{array}$} & \multirow[t]{2}{*}{$\mathrm{p}^{2}$} \\
\hline & Mediana & $95 \%$ IC & Mediana & $95 \%$ IC & \\
\hline Energía kcal & 1.402 & (982-1625) & 1.542 & $(1390-1743)$ & $<0,05$ \\
\hline Proteínas g & 49,7 & $(43,4-69,5)$ & 63,2 & $(56,6-71,3)$ & NS \\
\hline Grasas g & 35,2 & $(26,9-48,8)$ & 42,7 & $(39,2-48,4)$ & NS \\
\hline H. Carbono g & 186 & $(146,7-241,8)$ & 188 & $(166,6-210,3)$ & NS \\
\hline Fibra $g$ & 13,0 & $(10,7-17,1)$ & 17,2 & $(13,9-18,8)$ & NS \\
\hline Colesterol mg & 105 & (72-152) & 113 & (90-138) & NS \\
\hline Vit A ug & 488 & $(343-606)$ & 729 & $(631-905)$ & $<0,05$ \\
\hline Vit B1 mg & 1,2 & $(0,9-1,6)$ & 1,7 & $(1,4-1,8)$ & $<0,001$ \\
\hline Vit B2 mg & 1,1 & $(0,9-1,3)$ & 2,1 & $(1,9-2,3)$ & $<0,001$ \\
\hline Vit B6 mg & 1,3 & $(0,9-1,6)$ & 2,5 & $(2,3-2,8)$ & $<0,001$ \\
\hline Vit B12 ug & 1,6 & $(1,1-2,5)$ & 3,4 & $(2,8-4,5)$ & $<0,001$ \\
\hline Folatos ug & 676,6 & $(568,1767,6)$ & 912,6 & $(779,9-1040.1)$ & $<0,01$ \\
\hline Vit C mg & 53,7 & $(39,8-61,4)$ & 122,5 & $(108,9-137,3)$ & $<0,001$ \\
\hline Vit E mg & 7,6 & $(5,5-10,1)$ & 17,9 & $(16,5-20,7)$ & $<0,001$ \\
\hline Calcio mg & 415 & $(236,8-734,1)$ & 789,0 & $(691,4-840,2)$ & $<0,001$ \\
\hline Hierro mg & 10,8 & $(9,4-12,8)$ & 14,2 & $(12,2-15,0)$ & $<0,001$ \\
\hline Zinc mg & 5,6 & $(4,5-7,5)$ & 11,6 & $(11,2-12,5)$ & $<0,001$ \\
\hline
\end{tabular}

${ }^{1}$ Programa de Alimentación Complementaria del Adulto Mayor.

${ }^{2}$ Test de Kruskal Wallis. 
Tabla 4. M ediana de porcentaje de adecuación del aporte de energía y nutrientes de la alimentación complementada o no con PACAM con respecto a la recomendación

\begin{tabular}{|c|c|c|c|c|c|}
\hline \multirow[t]{2}{*}{ Nutrientes } & \multicolumn{2}{|c|}{$\begin{array}{l}\text { Alimentación sin } \\
\operatorname{PACAM}^{1}(n=27)\end{array}$} & \multicolumn{2}{|c|}{$\begin{array}{l}\text { Alimentación con } \\
\operatorname{PACAM}^{1}(\mathrm{n}=73)\end{array}$} & \multirow[b]{2}{*}{$\mathrm{p}^{2}$} \\
\hline & Mediana & $95 \%$ IC & Mediana & $95 \%$ IC & \\
\hline Energía kcal & 77,2 & $(51,2-93,6)$ & 86,4 & $(78,4-96,4)$ & 0,08 \\
\hline Proteínas g & 77,2 & $(64,2-108,5)$ & 94,5 & $(83,6-109,8)$ & NS \\
\hline Fibras $g$ & 65,7 & $(52,5-86,5)$ & 85,3 & $(65,9-95,1)$ & NS \\
\hline Vit A $\mu \mathrm{g}$ & 66,3 & $(48,3-86,4)$ & 98,3 & $(80,8-117,4)$ & $<0,05$ \\
\hline Vit B1 mg & 109,1 & $(82,9-143,1)$ & 141,8 & $(128,2-156,7)$ & $<0,05$ \\
\hline Vit B2 mg & 93,8 & $(72,8-115,4)$ & 173,8 & $(157,1-198,2)$ & $<0,001$ \\
\hline Vit B $6 \mathrm{mg}$ & 77,3 & $(64,4-101,5)$ & 156,0 & $(140,8-168,6)$ & $<0,001$ \\
\hline Vit B12 $\mu \mathrm{g}$ & 66,3 & $(44,5-104,4)$ & 142,9 & $(118,6-188,7)$ & $<0,001$ \\
\hline Folatos $\mu \mathrm{g}$ & 169,2 & $(142,0-191,9)$ & 228,2 & $(194,9-260,0)$ & $<0,001$ \\
\hline Vit C mg & 70,8 & $(45,6-81,8)$ & 150,1 & $(138,0-168,2)$ & $<0,001$ \\
\hline Vit E mg & 50,7 & $(36,3-67,5)$ & 119,3 & $(109,7-137,9)$ & $<0,001$ \\
\hline Calcio mg & 34,6 & $(19,7-61,2)$ & 65,8 & $(57,6-70,0)$ & $<0,001$ \\
\hline Hierro mg & 133,8 & $(106,9-158,8)$ & 177,1 & $(152,8-187,5)$ & $<0,001$ \\
\hline Zinc mg & 68,1 & $(54,8-85,0)$ & 137,3 & $(117,1-142,6)$ & $<0,001$ \\
\hline
\end{tabular}

${ }^{1}$ Programa de Alimentación Complementaria del Adulto Mayor.

${ }^{2}$ Test de Kruskal Wallis.

Tabla 5. M ediana de porcentajes de aporte del PAC AM a la alimentación diaria según su consumo

\begin{tabular}{|lccccc|}
\hline Nutrientes & $\begin{array}{c}\mathrm{BLAD}^{1} \\
(\%)\end{array}$ & $\begin{array}{c}\mathrm{CAD}^{2} \\
(\%)\end{array}$ & $\begin{array}{c}\text { BLAD + CAD } \\
(\%)\end{array}$ & $\begin{array}{c}\text { Promedio } \\
(\%)\end{array}$ & $\mathrm{P}^{3}$ \\
& $\mathrm{~N}=33$ & $\mathrm{~N}=28$ & $\mathrm{~N}=100$ & \\
\hline Energía Kcal. & 5,8 & 10,2 & 17,3 & 8,7 & $<0,001$ \\
Proteínas g & 7,2 & 10 & 22,4 & 7,8 & $<0,001$ \\
H Carbono g & 7,8 & 13,2 & 24,7 & 9,1 & $<0,001$ \\
Grasas g & 5,9 & 11 & 22,7 & 6,9 & $<0,001$ \\
Fibras g & 1,8 & 25,6 & 25 & 2,1 & $<0,001$ \\
Colesterol mg & 1,7 & 1,8 & 5 & 1,5 & $<0,001$ \\
Vit A ug & 29,1 & 39,8 & 44,1 & 27,7 & $<0,001$ \\
Vit B1 UL & 17,4 & 15,2 & 33,8 & 30,4 & $<0,001$ \\
Vit B2 mg & 22,9 & 11,3 & 28,6 & 9,9 & $<0,001$ \\
Vit B 6 mg & 13,5 & 15,5 & 29,1 & 13,8 & $<0,001$ \\
Vit B12 ug & 20,8 & 23,6 & 34,7 & 15,9 & $<0,001$ \\
Folatos ug & 11,4 & 6,5 & 19,5 & 11,9 & $<0,001$ \\
Vit C mg & 30,3 & 20,1 & 54,2 & 27,6 & $<0,001$ \\
Vit E mg & 41,4 & 27,6 & 60,4 & 36,7 & $<0,001$ \\
Calcio mg & 49,5 & 19,9 & 66,9 & 42,5 & $<0,001$ \\
Hierro mg & 40,6 & 34,4 & 66,9 & 38,5 & $<0,001$ \\
Zinc mg & 12,0 & 18,2 & 33,7 & 12,5 & $<0,001$ \\
\hline
\end{tabular}

${ }^{1}$ Crema Años Dorados. ${ }^{2}$ Bebida Láctea Años Dorados. ${ }^{3}$ Test de Kruskal Wallis. 
mayor consumo por otros integrantes de la familia. Los alimentos especialmente formulados tienen un mayor aporte de micronutrientes, pero el gran riesgo es la fatiga por consumo crónico.

Teniendo en cuenta que la "Crema Años Dorados" ha sido entregada desde hace más de 5 años, es posible esperar que su consumo habitual cause fatiga o aburrimiento $y$, por ende, una menor ingesta. Dado que su preparación generalmente está incluida en comidas destinadas a todos los miembros del hogar, ello podría determinar una mayor dilución intrafamiliar. Pese a ello, la valoración de la "Crema Años Dorados" sigue siendo bastante favorable por parte de los adultos mayores, destacando que todavía, aproximadamente, un tercio de los beneficiarios la consume diariamente.

Destacó una muy buena aceptabilidad de la bebida láctea por parte de los beneficiarios, refrendando los resultados obtenidos en el estudio piloto $^{13}$. Ello se vio reflejado por el mayor número de retiros mensuales desde su incorporación al PACAM, mayor frecuencia de consumo semanal, mayor número de raciones diarias y menor dilución intrafamiliar. Los casos de irregularidad en los retiros de los alimentos del programa, en su mayoría estuvieron relacionados con viajes fuera de la ciudad (meses de verano), motivos laborales o de salud. La entrega gratuita de la bebida láctea significó además un ahorro monetario en la compra de leche, que fue positivamente valorado por los beneficiarios. También incentivó a un mayor consumo de leche, debido a que anteriormente por imposibilidad económica en esta población, dicho alimento no era de consumo habitual.

No existen evaluaciones integrales sobre el impacto del programa de alimentación complementaria del adulto mayor en Chile, salvo un efecto positivo sobre los niveles de zinc de la población, intervenida después de tres meses de consumo ${ }^{14} \mathrm{y}$ en la funcionalidad, especialmente cuando se asocia a actividad física ${ }^{15}$. Otros estudios demuestran también que un aporte nutricional adecuado en dicha población puede mejorar el estado nutricional y los niveles deficientes de micronutrientes, reduciendo la incidencia de varias enfermedades crónicas y contribuyendo a su vez a reducir los costos en la atención de salud ${ }^{16-22}$.

Indudablemente el PACAM representa un medio muy favorable de complementación de micro- nutrientes. Los importantes niveles de deficiencias descritos previamente han disminuido en forma considerable, en parte, probablemente, por una mejor calidad de la alimentación del hogar, pero también por el significativo aporte que realizan los alimentos del programa. De los resultados del estudio destaca que cuando se incorporan en la alimentación ambos suplementos, ellos aportan más de 50\% del consumo total diario de algunos micronutrientes.

Llama la atención, sin embargo, que en muchos nutrientes, especialmente vitaminas del complejo $\mathrm{B}$, se sobrepasa la recomendación, alcanzando en el caso del folato adecuaciones superiores a 200\%. Dado que el nivel máximo de tolerancia es relativamente bajo para este nutriente (2,5 veces la recomendación), el buen aporte que hace la harina de trigo y la existencia de algunos estudios que asocian un mayor consumo de folatos con riesgo de cáncer de colon, es necesario revisar el nivel de fortificación para este nutriente ${ }^{23}$.

Destaca un bajo grado de adecuación del consumo energético en el grupo estudiado, a pesar de que una fracción importante del grupo tiene un IMC sobre lo normal. Esta situación, que ha sido descrita en otros estudios, sugiere la posibilidad de un subregistro del consumo real o una sobreestimación de los requerimientos, en una población que es mayoritariamente sedentaria ${ }^{3,4}$.

Los resultados de este estudio han corroborado que la bebida láctea presenta una excelente aceptabilidad, tolerancia y consumo por parte de los beneficiarios desde su incorporación al programa. Sin embargo, aún no se ha evaluado el impacto nutricional del PACAM, lo que es fundamental para analizar su costo-efectividad y decidir eventuales ampliaciones o modificaciones. Aun cuando el diseño de un estudio de impacto es complejo, por la falta de un adecuado grupo control, es imperativo su realización con el propósito de evaluar el efecto sobre el estado nutricional, incluyendo niveles plasmáticos de micronutrientes, funcionalidad, inmunidad, o grado de control o prevalencia de enfermedades crónicas, de acuerdo a los objetivos planteados por el programa. Sólo así se podrá establecer si la inversión actual, que supera los US\$19 millones al año, se justifica no sólo por razones sociales sino además sanitarias y económicas. 


\section{REFERENCIAS}

1. Castillo C, Uauy R, Atalah E, eds. Guías de alimentación para el adulto mayor: bases para la acción. Santiago: Imprenta Diario La Nación, 1999.

2. Instituto Nacional de Estadística. Chile: estimaciones y proyecciones de población. Disponible en: http://www.ine.cl/canales/chile_estadistico/ demografia_y_vitales/demografia/demografia.php (consultado el 23 de julio 2007).

3. Atalah E, Benavides $\mathrm{X}$, Ávila L, Barahona S, Cárdenas S. Características alimentarias de adultos mayores de comunas pobres de la Región Metropolitana. Rev Méd Chile 1998; 126: 489-96.

4. Castillo O, Rozowski J, Cuevas A, Maiz A, Soto M, Mardones F, Leighton F. Ingesta de nutrientes en adultos mayores de la comuna de Providencia, Santiago de Chile. Rev Méd Chile 2002; 130: 1335-42.

5. Urteaga C, Ramos R, Atalah E. Validación del criterio de evaluación nutricional global del adulto mayor. Rev Méd Chile 2001; 129: 871-6.

6. Ministerio de Salud de Chile. Crema Años Dorados: especificaciones técnicas y control de calidad, Santiago junio 2006. Disponible en: http:// www.minsal.cl/ici/nutricion/nutricion.html (consultado el 23 julio 2007)

7. Ministerio de Salud de Chile. Bebida Láctea Años Dorados: especificaciones técnicas y control de calidad, Santiago agosto 2005. Disponible en: http: //www.minsal.cl/ici/nutricion/Bebida_lactea_SIN_omega.pdf [consultado 23 julio 2007].

8. FAO. Human energy requirement. Report of a $\mathrm{FAO} / \mathrm{WHO} / \mathrm{UNU}$ expert consultation. Rome, October 2001

9. Institute of Medicine of the National Academies. Dietary Reference Intake: guiding principles for nutrition labeling and fortification. The National Academies Press, Washington DC, 2004

10. Atalah E, Benavides X, Cárdenas R, Barahona S, Espinoza M. Aceptabilidad y consumo de un suplemento alimentario en el adulto mayor. Rev Chil Nutr 2001; 28: 438-45.

11. Caballero E, Atalah E. Evaluación de la Aceptabilidad y Consumo de un suplemento alimentario en la República de Panamá. Rev Chil Nutr 2003; 30: 133-40.

12. Rosado JL, Rivera J, López G, Solano L, Rodríguez G, Casanueva E, García A. Desarrollo y evaluación de suplementos alimenticios para el Programa de Educación, Salud y Alimentación. Salud Pública Mex 1999; 41: 153-62.

13. Vera G, Alviña M, Araya H, Mizon C, Atalah E. Desarrollo de la bebida láctea Años Dorados, destinada al PACAM: informe final. Departamento de Nutrición, Facultad de Medicina, Universidad de Chile. Santiago 2003.

14. Ramos Ri, Atalah E, Urteaga C, Castañeda R, Orozco M, Ávila L, Martínez H. Efecto del consumo de un complemento alimentario en la concentración plasmática de cinc en adultos mayores residentes en Santiago de Chile. Rev Méd Chile 2007: 135: 1015-24.

15. Bunout D, Barrera G, De la Maza P, Avendano M, Gattas V, Peterman M et al. The Impact of Nutritional Supplementation and Resistance Training on the Health Functioning of Free-Living Chilean Elders: Results of 18 Months of Followup. J Nutr 2001; 131: 2441-6.

16. Arnaud-Battandier F, Malvy D, Jeandel C, Schmitt C, Aussage P, Beaufrere B, Cynober L. Use of oral supplements in malnourished elderly patients living in the community: a pharmaco-economic study. Clin Nutr 2004; 23: 1096-103.

17. Mcalpine SJ, Harper J, Mcmurdo ME, Bolton-Smith C, Hetherington MM. Nutritional supplementation in older adults: pleasantness, preference and selection of sip-feeds. Br J Health Psychol 2003; 8: 57-66.

18. Mckay DL, Perrone G, Rasmussen H, Dallal G, Hartman W, CaO G. et al. The effects of a multivitamin/mineral supplement on micronutrient status, antioxidant capacity and cytokine production in healthy older adults consuming a fortified diet. J Am Coll Nutr 2000; 19: 613-21.

19. Millen BE, Ohls JC, Ponza M, Mc Cool AC. The elderly nutrition program: an effective national framework for preventive nutrition interventions. J Am Diet Assoc 2002; 102: 234-40.

20. Acosta RS, Fernández K, Fusta M. Evaluation of a state social program including a nutritional component implemented in the city of Cordoba for the elderly. 1999. Rev Fac Cien Med Univ Nac Cordoba 2000; 57: 199-217.

21. Barr SI, Mccarron Da, Heaney RP, Dawson-Hughes B, Berga SL, Stern JS et al. Effects of increased consumption of fluid milk on energy and nutrient intake, body weight, and cardiovascular risk factors in healthy older adults. J Am Diet Assoc 2000; 100: 810-7.

22. Krondl M, Coleman PH, Bradley Cl, Lau D, Ryan N. Subjectively healthy elderly consuming a liquid nutrition supplement maintained body mass index and improved some nutritional parameters and perceived well-being. J Am Diet Assoc 1999; 99: $1542-8$.

23. Pellis L, Dommels Y, Venema D, Polanen Av, Lips E, BAYKUS H ET AL. High folic acid increases cell turnover and lowers differentiation and iron content in human HT29 colon cancer cells. Br J Nutr 2007; 98: 1-6. 